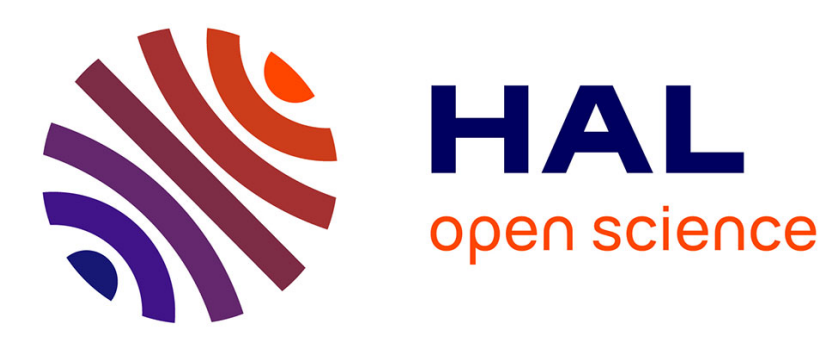

\title{
3-Colorable Planar Graphs Have an Intersection Segment Representation Using 3 Slopes
}

Daniel Gonçalves

\section{To cite this version:}

Daniel Gonçalves. 3-Colorable Planar Graphs Have an Intersection Segment Representation Using 3 Slopes. WG 2019 - 45th International Workshop on Graph-Theoretic Concepts in Computer Science, Jun 2019, Vall de Núria, Spain. pp.351-363, 10.1007/978-3-030-30786-8_27 . lirmm-02407838

\section{HAL Id: lirmm-02407838 https://hal-lirmm.ccsd.cnrs.fr/lirmm-02407838}

Submitted on 13 Dec 2019

HAL is a multi-disciplinary open access archive for the deposit and dissemination of scientific research documents, whether they are published or not. The documents may come from teaching and research institutions in France or abroad, or from public or private research centers.
L'archive ouverte pluridisciplinaire HAL, est destinée au dépôt et à la diffusion de documents scientifiques de niveau recherche, publiés ou non, émanant des établissements d'enseignement et de recherche français ou étrangers, des laboratoires publics ou privés. 


\title{
3-colorable planar graphs have an intersection segment representation using 3 slopes $^{\star}$
}

\author{
Daniel Gonçalves \\ LIRMM, Université de Montpellier \& CNRS, France.
}

\begin{abstract}
In his $\mathrm{PhD}$ Thesis E.R. Scheinerman conjectured that planar graphs are intersection graphs of segments in the plane. This conjecture was proved with two different approaches. In the case of 3-colorable planar graphs E.R. Scheinerman conjectured that it is possible to restrict the set of slopes used by the segments to only 3 slopes. Here we prove this conjecture by using an approach introduced by S. Felsner to deal with contact representations of planar graphs with homothetic triangles.
\end{abstract}

Keywords: Planar graphs $\cdot$ Segment intersections.

\section{Introduction}

In this paper, we consider intersection representations for planar graphs. A segment representation of a graph $G$ maps every vertex $v \in V(G)$ to a segment $\mathbf{v}$ of the plane so that two segments $\mathbf{u}$ and $\mathbf{v}$ intersect if and only if $u v \in E(G)$. Although this graph family is simply defined, it is not easy to manipulate. Actually, even if this class of graphs is small (there are less than $2^{O(n \log n)}$ such graphs with $n$ vertices [16]) a segment representation may be long to encode (in the representations of some of these graphs the endpoints of the segments need at least $2^{\sqrt{n}}$ bits to be coded [14]). There are also interesting open problems concerning this class of graphs. For example, we know that deciding whether a graph $G$ admits a segment representation is NP-hard, indeed it is $\exists \mathbb{R}$-complete [12] but it is still open whether this problem belongs to NP or not. Here we focus on segment representations for planar graphs.

In his PhD Thesis, E.R. Scheinerman [17] conjectured that every planar graph has a segment representation. This conjecture attracted a lot of attention. H. de Fraysseix and P. Ossona de Mendez [7] proved it for a large family of planar graphs, the planar graphs having a 4-coloring in which every induced cycle of length 4 uses at most 3 colors. In particular, this implies the conjecture for 3-colorable planar graphs. Then J. Chalopin and the author finally proved this conjecture [2]. Recently, a much simpler proof was provided by the author, L. Isenmann, and C. Pennarun [9]. Here we focus on segment representations of planar graphs with further restrictions.

In his PhD Thesis, E.R. Scheinerman [17] proved that every outerplanar graph has a segment representation where only 3 slopes are used, and where parallel segments do not intersect. Let us call such a representation a 3-slopes segment representation. This result led E.R. Scheinerman conjecture [18] (see also [7]) that such representation

\footnotetext{
* This research is partially supported by the ANR GATO, under contract ANR-16-CE40-0009.
} 
exists for every 3-colorable planar graph. Later, several groups proved a related result on bipartite planar graphs $[3,8,11]$. They proved that every bipartite planar graph has a 2-slopes segment representation, with the extra property that segments do not cross each other. Let us call such a representation a 2-slopes contact segment representation. More recently de Castro et al. [1] considered a particular class of 3-colorable planar graphs. They proved that every triangle-free planar graph has a 3-slopes contact segment representation. Such a contact segment representation cannot be asked for any 3-colorable planar graph. Indeed, up to isomorphism, the octahedron has only one 3slopes contact segment representation depicted in Figure 1, and one can easily check that this representation does not extend to the (3-colorable) graph obtained after gluing a copy of an octahedron in each of its faces. However, we will use 3-slopes contact segment representations in the proof of our main result.

Theorem 1. Every 3-colored planar graph has a 3-slopes segment representation such that parallel segments correspond to the color classes.
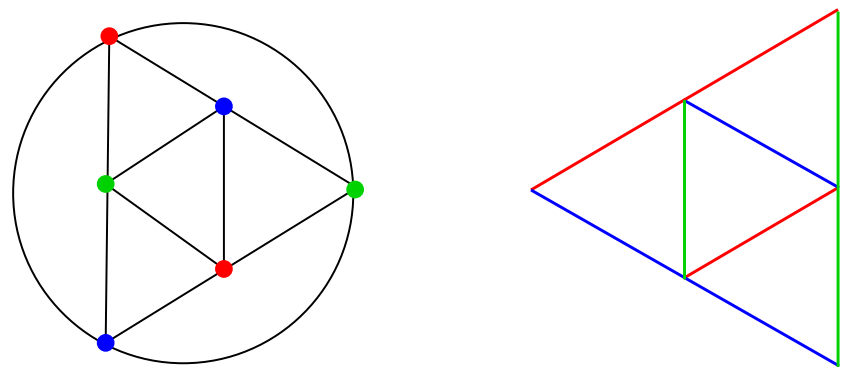

Fig. 1. The octahedron and a 3-slopes contact representation. It is unique, up to vertex automorphism, up to scaling, and once the slopes are set.

As every 3-colored planar graph is the induced subgraph of some 3-colored triangulation we only consider the case of triangulations in the following. In Section 2 we review some basic definitions. Section 3 is devoted to the so-called triangular contact schemes. It is shown that every 3-colorable triangulation admits such a scheme. Then, those schemes are used in Section 4 to build 3-slopes segment representations. Finally, we conclude with some remarks on 4-slopes segment representations.

\section{Terminology}

A triangulation is a plane graph where every face has size three. Unless stated otherwise, in this paper triangulations are simple, that means without loops nor multiple edges. A triangulation $T$, simple or not, is Eulerian if every vertex has even degree. It is folklore that these triangulations are the 3-colorable triangulations. Actually these triangulations are uniquely 3 -colorable (up to color permutation). Hence their vertex 
set $V(T)$ is canonically partitioned into three independent sets $A, B$ and $C$. In the following we will denote the vertices of these sets respectively $a_{i}$ with $0 \leq i<|A|, b_{j}$ with $0 \leq j<|B|$, and $c_{k}$ with $0 \leq k<|C|$. In such a triangulation $T$ any face is incident to one vertex $a_{i}$, one vertex $b_{j}$, and one vertex $c_{k}$, and these vertices appear in this order either clockwisely or counterclockwisely. In the following, the vertices of the outerface are always denoted $a_{0}, b_{0}$ and $c_{0}$, and they appear clockwisely in this order around $T$. As the orders of two adjacent faces are opposite, the dual graph of $T$ is bipartite. Given an Eulerian triangulation $T$ with face set $F(T)$, let us denote by $F_{1}(T)$ and $F_{2}(T)$ (or simply $F_{1}$ and $F_{2}$ if it is clear from the context) the face sets partitioning $F(T)$, such that no two adjacent faces belong to the same set, and such that $F_{2}(T)$ contains the outer-face. Note that by construction for any face $f \in F_{1}(T)$ (resp. $f \in F_{2}(T)$ ) its vertices $a_{i}, b_{j}$ and $c_{k}$ appear in clockwise (resp. counterclockwise) order around $f$. Note that the vertices $a_{0}, b_{0}$ and $c_{0}$ appear in clockwise order around $T$, but in counterclockwise order w.r.t. the outer face. Let $n=|V(T)|$. As $T$ is a triangulation, by Euler's formula it has $2 n-4$ faces. Hence, as $T$ 's dual is bipartite and 3-regular, $\left|F_{1}(T)\right|=\left|F_{2}(T)\right|=n-2$.

In the following we build 3 -slopes segment representations. The 3 slopes used are expected to be distinct, but apart from that the exact 3 slopes considered do not matter. Indeed, for any two triples of slopes, $\left(s_{1}, s_{2}, s_{3}\right)$ and $\left(s_{1}^{\prime}, s_{2}^{\prime}, s_{3}^{\prime}\right)$, there exists an affine map of the plane turning any 3-slopes segment representation using slopes $\left(s_{1}, s_{2}, s_{3}\right)$ into a 3 -slopes segment representation using slopes $\left(s_{1}^{\prime}, s_{2}^{\prime}, s_{3}^{\prime}\right)$. We denote $\vec{a}, \vec{b}$, and $\vec{c}$ the vectors corresponding to slopes of the sets $A, B$, and $C$ respectively. The magnitude of these vectors is chosen such that $\vec{a}+\vec{b}+\vec{c}=\overrightarrow{0}$.

\section{TC-representations and TC-schemes}

We begin with the definition of particular 3-slopes contact representations illustrated in Figure 2.

Definition 1. $A$ Triangular 3-slopes segment Contact representation (TC-representation for short) is a 3-slopes contact segment representation using the same slopes as $\vec{a}, \vec{b}$, and $\vec{c}$, and where:

- Three segments $\mathbf{a}_{0}, \mathbf{b}_{0}$, and $\mathbf{c}_{\mathbf{0}}$, form a triangle which contains all the other segments.

- Every inner region is a triangle, whose each side is contained in a segment of the representation.

- Two parallel segments intersect on at most one point, their endpoint.

Definition 2. Let the plane graph $M(\mathcal{R})$ induced by a TC-representation $\mathcal{R}$ be the map whose vertices correspond to the segments of the representation, and where two vertices are adjacent if and only if the corresponding segments form a corner of one of the inner triangles. The orders of the neighbors around a vertex $v$ correspond to the order of the segments around $\mathbf{v}$. 

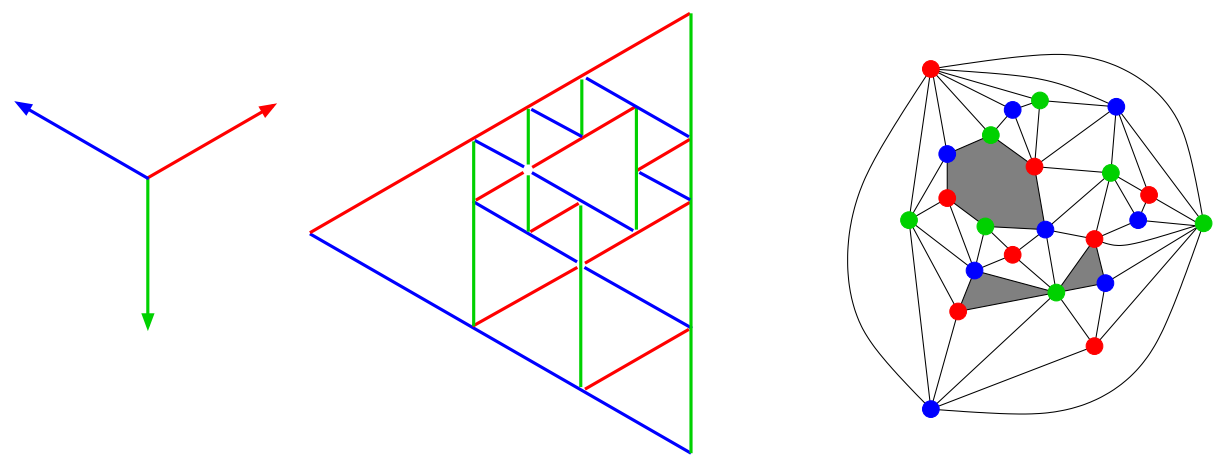

Fig. 2. (left) Vectors $\vec{a}, \vec{b}$, and $\vec{c}$. (middle) A TC-representation with various types of intersection points. (right) Its induced graph, where gray faces are particular degenerate faces. One has size six, and there are two faces of size three that correspond to the same intersection point.

Note that the plane graph induced by a TC-representation has several properties. For example, two parallel segments correspond to non-adjacent vertices. The slopes hence define a 3-coloring of the graph. Note also that the dual graph of $M(\mathcal{R})$ is bipartite. Indeed such a map has two types of faces, one set contains the (triangular) faces corresponding to the inner regions of the TC-representation, and the other set contains the outerface and the faces corresponding to intersection points. Let us denote the latter faces degenerate faces, and note that those faces have size three or six. A size six face $\left(a_{i}, b_{j}, c_{k}, a_{i^{\prime}}, b_{j^{\prime}}, c_{k^{\prime}}\right)$ comes from the intersection point of six segments, and as those six segments go in distinct directions they do not intersect elsewhere, so this cycle has no chord in $M(\mathcal{R})$. Finally note that going clockwise in any inner region one successively follows $\alpha \vec{a}, \alpha \vec{b}$, and then $\alpha \vec{c}$, for some not necessarily positive value $\alpha$.
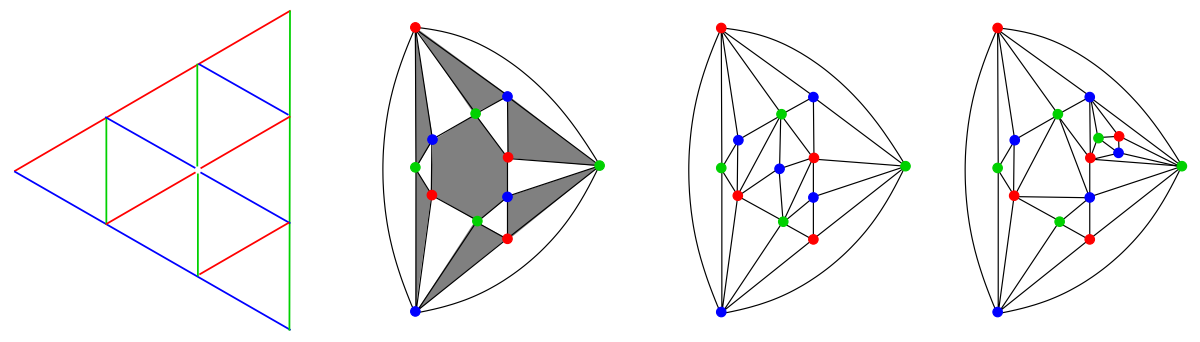

Fig. 3. From left to right. A TC-representation $\mathcal{R}$; its induced map $M(\mathcal{R})$, where gray faces are the degenerate faces; and two triangulations having $\mathcal{R}$ as TC-scheme. 
Definition 3. A TC-representation $\mathcal{R}$ is a TC-scheme of an Eulerian triangulation $T$ if $M(\mathcal{R})$ is a subgraph of $T$ with the same outer-face as $T$ and such that the vertices and edges of $V(T) \backslash V(M(\mathcal{R}))$ lie inside degenerate faces of $M(\mathcal{R})$ (see Figure 3).

Actually as in $M(\mathcal{R})$, the inner faces around any vertex alternate among degenerate and non-degenerate. This implies that every edge of $M(\mathcal{R})$ bounds a non-degenerate face, and a face that is degenerate or that is the outerface. We thus have the following.

Remark 1. A TC-representation $\mathcal{R}$ is a TC-scheme of $T$ if and only if the non-degenerate faces of $M(\mathcal{R})$ and its outerface are faces of $T$.

The main ingredient in the proof of Theorem 1 is the following.

Theorem 2. Every Eulerian triangulation $T$ has a TC-scheme, and this scheme is unique.

To prove this theorem we proceed by the following steps. We first model TCschemes of $T$ by means of a system of linear equations and we sketch out why this linear system always has a unique solution.

\subsection{The linear system model}

In a TC-representation all the triangles are homothetic. Let us define the size of a triangle as its relative size with respect to the outer-triangle. We may require that the outer-triangle has size 1 , the triangles with a corner on the left have positive sizes, while the triangles with a corner on the right have negative sizes. The variables of our linear system correspond to the sizes of the triangular regions. So for each face $f \in F_{1}$ we have a variable $x_{f}$. Informally, the value of $x_{f}$ will prescribe the size and shape of the corresponding triangle in a TC-representation. If $x_{f}<0, x_{f}=0$, or if $x_{f}>0$ the corresponding triangle has a corner on the right, is missing, or has a corner on the left, respectively.

Let us denote by $F_{1}(v)$ the subset of faces of $F_{1}$ incident to $v$. As the outer triangle has size 1 and contains the other triangles, the faces in $F_{1}\left(a_{0}\right)$ should have non-negative sizes, and they should sum up to 1 (see Figure 4 , left). We hence consider the following constraint.

$$
\sum_{f \in F_{1}\left(a_{0}\right)} x_{f}=1
$$

We add no constraint about the sign of these sizes. Note that similar constraints hold for $b_{0}$ and $c_{0}$.

$$
\begin{gathered}
\sum_{f \in F_{1}\left(b_{0}\right)} x_{f}=1 \\
\sum_{f \in F_{1}\left(c_{0}\right)} x_{f}=1
\end{gathered}
$$

Similarly, around an inner segment of a TC-representation all the triangles on one side have same size sign, which is opposite to the other side. Furthermore, by summing 
all these sizes one should obtain 0 (see Figure 4, right). Hence, for any inner-vertex $u$ we consider the following constraint.

$$
\sum_{f \in F_{1}(u)} x_{f}=0
$$
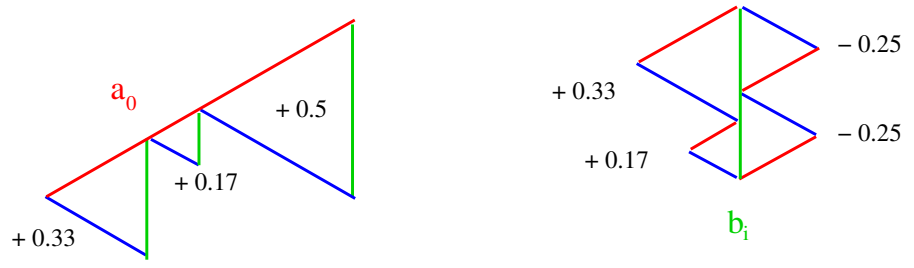

Fig. 4. (left) The size of the triangles around $a_{0}$. (right) The size of the triangles around some inner vertex $b_{i}$.

In the following, Equation $\left(a_{j}\right)$ will refer to Equation $(u)$ where vertex $u$ is replaced by $a_{j}$. Note that every face $f \in F_{1}$ is incident to exactly one vertex of $A$, one vertex of $B$, and one vertex of $C$. Hence by summing Equations $\left(a_{0}\right),\left(a_{1}\right), \ldots,\left(a_{|A|}\right)$, one obtains that $\sum_{f \in F_{1}} x_{f}=1$. The same holds with Equations $\left(b_{0}\right),\left(b_{1}\right), \ldots,\left(b_{|B|}\right)$, or with Equations $\left(c_{0}\right),\left(c_{1}\right), \ldots,\left(c_{|C|}\right)$. Equations $\left(b_{0}\right)$ and $\left(c_{0}\right)$ are hence implied by the others and thus we do not need to consider them anymore. Let us denote by $\mathcal{L}$ the obtained system of $n-2$ linear equations on $\left|F_{1}\right|=n-2$ variables.

Let us define the set $V^{\prime}=V \backslash\left\{b_{0}, c_{0}\right\}$ of size $n-2$. Finding a solution to $\mathcal{L}$ is equivalent to finding a vector $S \in \mathbb{R}^{F_{1}}$ (that is a vector indexed by elements of $F_{1}$ ) such that $M S=I$, where $M \in \mathbb{R}^{V^{\prime} \times F_{1}}$ (a square matrix indexed by elements of $V^{\prime} \times F_{1}$ ) and $I \in \mathbb{R}^{V^{\prime}}$ are defined by

$$
M\left(x_{i}, f\right)=\left\{\begin{array}{ll}
1 & \text { if } f \in F_{1}\left(x_{i}\right) \\
0 & \text { otherwise. }
\end{array} \quad I\left(x_{i}\right)= \begin{cases}1 & \text { if } x_{i}=a_{0} \\
0 & \text { otherwise }\end{cases}\right.
$$

Given some bijective mappings $g_{V^{\prime}}:[1, \ldots, n-2] \longrightarrow V^{\prime}$ and $g_{F_{1}}:[1, \ldots, n-$ $2] \longrightarrow F_{1}$, one can index the elements of $M$ by pairs $(i, j) \in[1, \ldots, n-2] \times[1, \ldots, n-$ 2 ], and thus define the determinant of $M$. By the following lemma, $\mathcal{L}$ has a solution vector $S$, and this solution is unique.

Lemma 1. The matrix $M$ is non-degenerate, i.e. $\operatorname{det}(M) \neq 0$.

The full proof of this lemma is inspired by the work of Felsner [4] on contact representations with homothetic triangles. The proof is not provided in this extended abstract but the main idea is to consider the bipartite graph $T_{M}$ with independent sets $V^{\prime}$ and $F_{1}$ such that $v \in V^{\prime}$ and $f \in F_{1}$ are adjacent if and only if $v$ and $f$ are incident in $T$. Note that $M$ is the biadjacency matrix of $T_{M}$. From the embedding of $T$ one can easily 
embed $T_{M}$ in such a way that all the inner faces have size 6 , and such that $a_{0}$ is on the outerboundary.

Note that every perfect matching of $T_{M}$ (if any) corresponds to a permutation $\sigma$ on $[1, \ldots, n-2]$ (we say $\sigma$ belongs to the permutation group $S_{n-2}$ ) defined by $\sigma\left(g_{F_{1}}^{-1}(f)\right)=g_{V^{\prime}}^{-1}(v)$, for any edge $v f$ of the perfect matching. If the obtained permutation is even we call such perfect matching positive, otherwise it is negative. From the Leibniz formula for the determinant,

$$
\operatorname{det}(M)=\sum_{\sigma \in S_{n-2}} \operatorname{sgn}(\sigma) \prod_{i \in[1, \ldots, n-2]} M\left(g_{V^{\prime}}(\sigma(i)), g_{F_{1}}(i)\right)
$$

, one can see that $\operatorname{det}(M)$ counts the number of positive perfect matchings of $T_{M}$ minus its number of negative perfect matchings.

Claim. The graph $T_{M}$ admits at least one perfect matching.

Given a graph $G$ and a perfect matching $M$ of $G$, an alternating cycle $C$ is a cycle of $G$ with edges alternating between $M$ and $E(G) \backslash M$. Note that replacing in $M$ the edges of $M \cap C$ by the edges of $C \backslash M$ yields another perfect matching. We call such operation a cycle exchange. It is folklore that the set of perfect matchings of a graph are linked by cycle exchanges. Actually, for $T_{M}$ one can restrict itself to cycles of length six.

Claim. All the perfect matchings of $T_{M}$ are linked by 6 -cycle exchanges.

This implies that the perfect matchings of $T_{M}$ are either all positive, or all negative. Thus $\operatorname{det}(M) \neq 0$. The following lemma (not proved in this extended abstract) allows us to conclude the proof of Theorem 2 .

Lemma 2. Every Eulerian triangulation $T$ admits a TC-scheme $\mathcal{R}$ that corresponds to the solution of its linear system $\mathcal{L}$.

\section{3-slopes segment representations}

In this section we use Theorem 2 to prove the main theorem of the article, Theorem 1. As already mentioned it is sufficient to prove it for Eulerian triangulations. Given an Eulerian triangulation $T$, let us denote $a_{1}, b_{1}$ and $c_{1}$ the vertices forming a face with vertices $b_{0}$ and $c_{0}$, with $a_{0}$ and $c_{0}$, and with $a_{0}$ and $b_{0}$, respectively. Theorem 1 follows from the following technical proposition.

Proposition 1. For every $\epsilon>0$, every Eulerian triangulation $T$ admits a 3-slopes segment representations $\mathcal{R}$ such that:

- The segments $\mathbf{a}_{0}, \mathbf{b}_{0}$, and $\mathbf{c}_{\mathbf{0}}$ form a triangle $\Delta$ of size 1 (its sides are obtained by following $\vec{a}, \vec{b}$, and $\vec{c}$ ).

- Every segment is contained in the hexagon centered on $\Delta$, obtained by successively following $(1-\epsilon) \vec{a},-2 \epsilon \vec{c},(1-\epsilon) \vec{b},-2 \epsilon \vec{a},(1-\epsilon) \vec{c}$, and $-2 \epsilon \vec{b}$ (see Figure 5). 

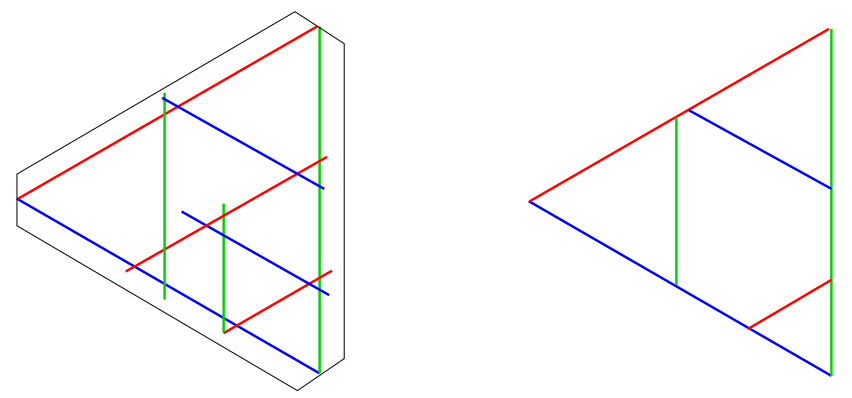

Fig. 5. (left) A 3-slopes segment representation inside an hexagon. (right) A scheme representing its shape.

Given such representation $\mathcal{R}$, we define the shape of $\mathcal{R}$ as the triplet $\left(s_{a}, s_{b}, s_{c}\right)$ of sizes in $\mathcal{R}$ (w.r.t. the outer-triangle) of the triangles corresponding to $a_{1} b_{0} c_{0}, a_{0} b_{1} c_{0}$, $a_{0} b_{0} c_{1}$, respectively. Note that if $\epsilon$ is chosen sufficiently small, that is for $\epsilon<1$, as the vertices $a_{1}, b_{1}$, and $c_{1}$ have neighbors that are inner vertices, $\mathbf{a}_{\mathbf{1}}, \mathbf{b}_{\mathbf{1}}$, and $\mathbf{c}_{\mathbf{1}}$ intersect $\Delta$, and we have $s_{a}>0, s_{b}>0$, and $s_{c}>0$.

Proof. We proceed by induction as we assume that the proposition holds for any Eulerian triangulation with less vertices. The initial case of this induction, when $|V(T)|=3$ clearly holds.

Given an Eulerian triangulation $T$ with at least four vertices, we consider a TCscheme $\mathcal{R}$ of $T$ (given by Theorem 2), and by successively resolving degenerate points (i.e. intersection points of at least three segments) from left to right, we eventually reach the sought representation. Here resolving means that the segments of a 3-degenerate point (resp. a 6-degenerate point) are moved to form a triangle (resp. a polygon) inside which we are going to draw a 3-slopes representation of the graph corresponding to this degenerate face of $M(\mathcal{R})$, this is possible by using the induction on this smaller graph. The degenerate points of $\mathcal{R}$ are resolved from left to right. This means that at a given stage of this process there is a vertical line (parallel with $\vec{b}$ ) $\mathcal{V}$ such that on its left there is no intersection point of three or more segments. This implies that on the left of $\mathcal{V}$ the representation handles some small perturbations: one can slightly move the segments without changing the intersections.

Let $\mathcal{V}$ be the leftmost vertical line containing degenerate points. We resolve those degenerate points by slightly moving segments on the left of or on $\mathcal{V}$, while maintaining the right side of the representation unchanged. We consider different cases according to the degenerate points on $\mathcal{V}$.

If $\mathcal{V}$ contains a 3-degenerate point $\mathbf{p}$ in the interior of a (vertical) segment $\mathbf{b}_{\mathbf{j}}$ and at the end of two segments $\mathbf{a}_{\mathbf{i}}$ and $\mathbf{c}_{\mathbf{k}}$ lying on the left of $\mathcal{V}$, the situation is rather simple. Move these segments a little to the left and slightly prolong them to intersect $\mathbf{b}_{\mathbf{j}}$ (see Figure 6). As there is no degenerate point on the left of $\mathcal{V}$ these moves can be done while maintaining the existing intersections and avoiding new intersections. If $a_{i} b_{j} c_{k}$ is 

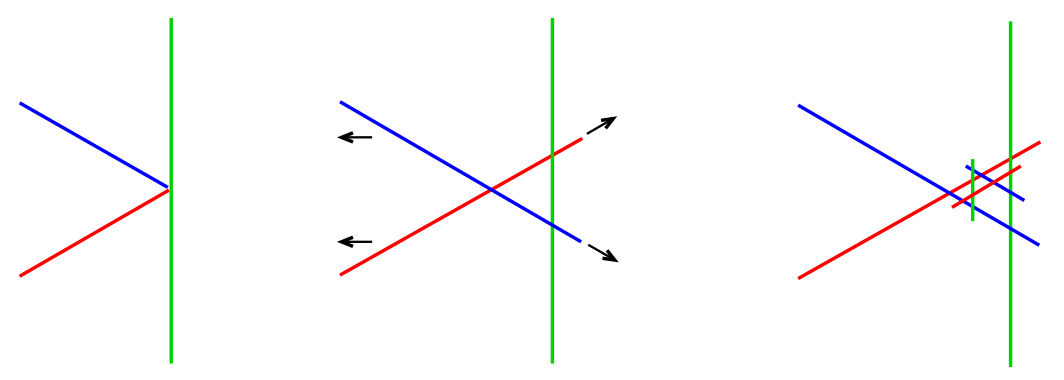

Fig. 6. (left) A 3-degenerate point on $\mathcal{V}$ (middle) Small perturbation of $\mathcal{R}$ (right) The addition of a representation inside the new triangle.

not a face of $T$, consider the triangulation $T^{\prime}$ induced by the vertices in the cycle $a_{i} b_{j} c_{k}$ of $T$. By induction $T^{\prime}$ has a representation that can be drawn inside the newly formed triangle bordered by the segments $\mathbf{a}_{\mathbf{i}}, \mathbf{b}_{\mathbf{j}}$ and $\mathbf{c}_{\mathbf{k}}$.
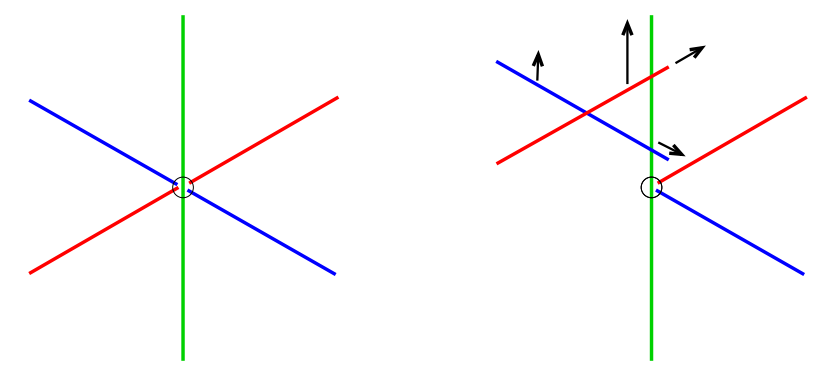

Fig. 7. (left) A double 3-degenerate point on $\mathcal{V}$ (right) Small perturbation of $\mathcal{R}$.

If $\mathcal{V}$ contains a double 3-degenerate point $\mathbf{p}$ in the interior of a (vertical) segment $\mathbf{b}_{\mathbf{j}}$, the situation is similar to the previous one. Move the segments on the left of $\mathcal{V}$ as depicted in Figure 7. If the new triangle is not a face of $T$, we add a representation inside. We are now left with a simple 3-degenerate point at $\mathbf{p}$. This corresponds to the following case.

If $\mathcal{V}$ contains a 3-degenerate point $\mathbf{p}$ in the interior of a (vertical) segment $\mathbf{b}_{\mathbf{j}}$ and at the end of two segments, $\mathbf{a}_{\mathbf{i}}$ and $\mathbf{c}_{\mathbf{k}}$, lying on the right of $\mathcal{V}$, one can move $\mathbf{b}_{\mathbf{j}}$ slightly to the right or slightly to the left and resolve these points without changing the right part of the representation. The choice of moving $b_{j}$ to the right or to the left is explained in the next paragraph, but we can assume this move to be arbitrarily small. Whatever the direction $\mathbf{b}_{\mathbf{j}}$ is moved one has to prolong $\mathbf{a}_{\mathbf{i}}$ and $\mathbf{c}_{\mathbf{k}}$ to have all the intersections, between these segments or with $\mathbf{b}_{\mathbf{j}}$ (see Figure 8). Note that in order to preserve the 

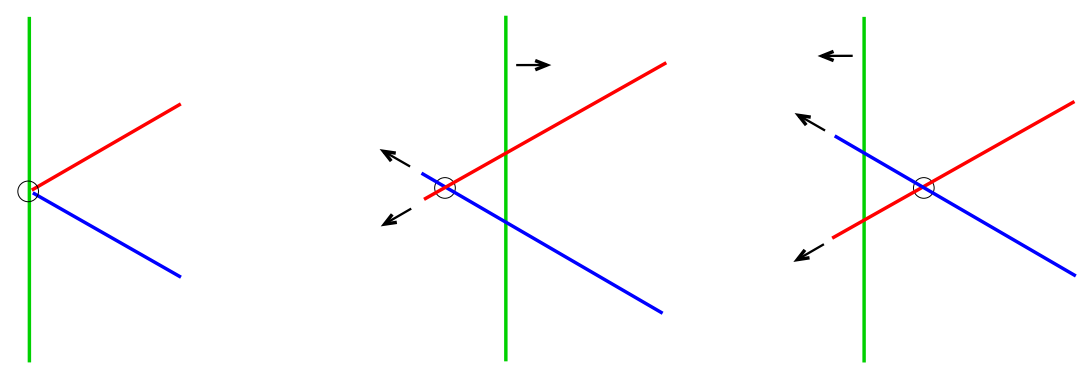

Fig. 8. (left) A 3-degenerate point on $\mathcal{V}$ (middle) Slightly moving $\mathbf{b}_{\mathbf{j}}$ to the right (right) Slightly moving $b_{j}$ to the left.

representation on the right of $\mathcal{V}$ the segments $\mathbf{a}_{\mathbf{i}}$ and $\mathbf{c}_{\mathbf{k}}$ are not moved, they are only prolonged around p. Again, if $a_{i} b_{j} c_{k}$ is not a face of $T$, we draw a representation inside the newly formed triangle. Note that if $b_{j}$ moves to the right, the triangle bordered by $\mathbf{a}_{\mathbf{i}}, \mathbf{b}_{\mathbf{j}}$ and $\mathbf{c}_{\mathbf{k}}$ has negative size, but it suffices to apply a homothety with negative ratio to obtain a representation that can be drawn inside.

Consider now the degenerate points at the end of a (vertical) segment $\mathbf{b}_{\mathbf{j}}$ of $\mathcal{V}$. Let $\mathbf{b}_{\mathbf{1}}, \mathbf{b}_{\mathbf{2}}, \ldots, \mathbf{b}_{\mathbf{t}}$ be a maximal sequence of segments on $\mathcal{V}$ such that $\mathbf{b}_{\mathbf{j}}$ and $\mathbf{b}_{\mathbf{j}+\mathbf{1}}$ intersect on a point. We are going to move these segments alternatively to the right and to the left, for example the segments with even index are moved to the left while the ones with odd index are moved to the right. The exact magnitude of these moves will be set later, but first note that the 3 -degenerate points in the interior of the segments $\mathbf{b}_{\mathbf{j}}$ with $1 \leq j \leq t$ can be dealt if the move of $\mathbf{b}_{\mathbf{j}}$ is sufficiently small (see previous cases). Consider the intersection point $\mathbf{p}$ between $\mathbf{b}_{\mathbf{j}}$ and $\mathbf{b}_{\mathbf{j}+\mathbf{1}}$. The case of $\mathbf{b}_{\mathbf{1}}$ and $\mathbf{b}_{\mathbf{t}}$ 's end is similar and it is not detailed here.
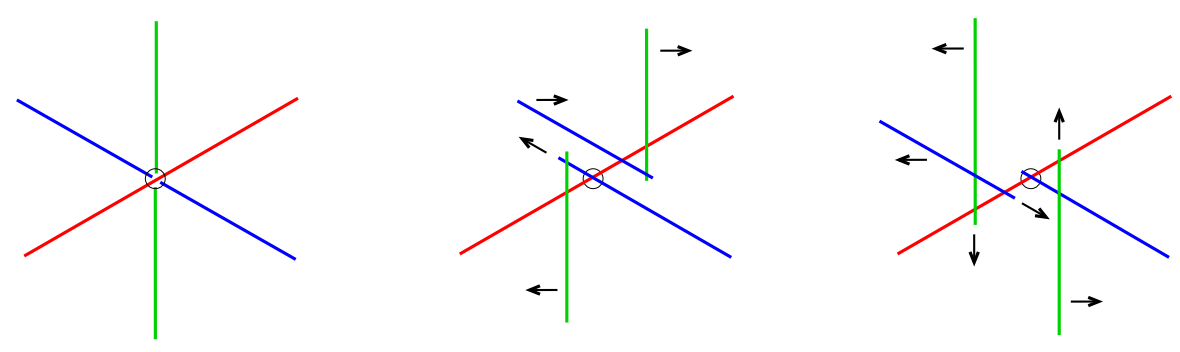

Fig. 9. (left) A double 3-degenerate point on $\mathcal{V}$ (middle) \& (right) Small moves that resolve this point.

If there is a segment $\mathbf{a}_{\mathbf{i}}$ going through $\mathbf{p}$. It is shown in Figure 9 how to resolve these two overlapped 3-degenerate points, in order to create two triangles, where one can add 
a small representation if needed. The case where there is a segment $\mathbf{c}_{\mathbf{k}}$ going through $\mathbf{p}$ is similar.
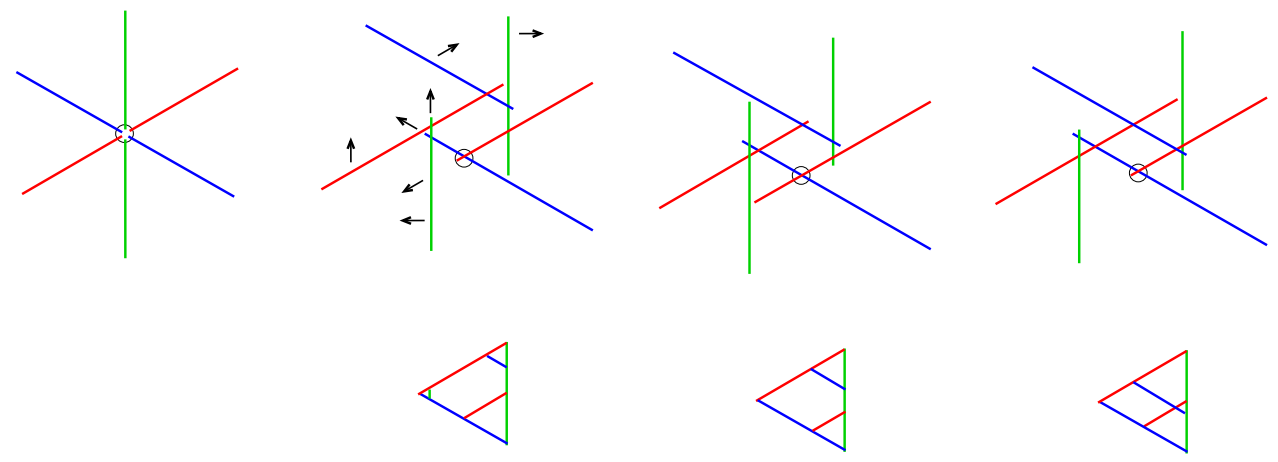

Fig. 10. From left to right : A 6-degenerate point on $\mathcal{V}$. Resolution if there is no chord in $b_{j} a c b_{j+1} a^{\prime} c^{\prime}$ with the shape of $\mathcal{R}^{\prime}$. Resolution if none of $b_{j} c, c a^{\prime}$, or $a^{\prime} b_{j}$ is a chord, with the shape of $\mathcal{R}^{\prime}$. Resolution if $a c^{\prime}$ and $c a^{\prime}$ are chords, with the shape of $\mathcal{R}_{2}$.

Assume now that six segments intersect at $\mathbf{p}$. Let $\mathbf{b}_{\mathbf{j}}$ be the one below $\mathbf{p}$, and let $\mathbf{a}, \mathbf{c}, \mathbf{b}_{\mathbf{j}+\mathbf{1}}, \mathbf{a}^{\prime}$, and $\mathbf{c}^{\prime}$ be the other ones around $\mathbf{p}$ clockwisely. Let us assume wlog that $\mathbf{b}_{\mathbf{j}}$ has to move to the left, while $\mathbf{b}_{\mathbf{j}+\mathbf{1}}$ has to move to the right. The degenerate face corresponding to $\mathbf{p}$ is bounded by these six vertices and there are several cases according to whether there are chords among them in $T$ (see Figure 10).

If there is no chord inside the cycle $b_{j} a c b_{j+1} a^{\prime} c^{\prime}$ we consider the subgraph of $T$ induced by the vertices on and inside this cycle, add we add the edges $a b_{j+1}, b_{j+1} c^{\prime}$, and $a c^{\prime}$ outside the cycle, and we denote by $T^{\prime}$ the obtained simple Eulerian triangulation. By the induction we know that $T^{\prime}$ admits a 3-slope segment representation $\mathcal{R}^{\prime}$, and let $\left(s_{a}, s_{b}, s_{c}\right)$ be the shape of $\mathcal{R}^{\prime}$. We resolve the point by moving the segments as depicted in Figure 10, and the magnitude of each of these moves is prescribed by the shape $\left(s_{a}, s_{b}, s_{c}\right)$ in order to allow us to copy $\mathcal{R}^{\prime}$ inside the triangle formed by $\mathbf{a}, \mathbf{b}_{\mathbf{j}+\mathbf{1}}$, and $\mathbf{c}^{\prime}$. Then we shorten $\mathbf{a}, \mathbf{b}_{\mathbf{j}+\mathbf{1}}$, and $\mathbf{c}^{\prime}$ to avoid intersections among them. Actually, the case where none of $a b_{j+1}, b_{j+1} c^{\prime}$, or $a c^{\prime}$ is a chord is similar.

If none of $b_{j} c, c a^{\prime}$, or $a^{\prime} b_{j}$ is a chord of $b_{j} a c b_{j+1} a^{\prime} c^{\prime}$ we proceed similarly. The only difference is that we add the edge $b_{j} c, c a^{\prime}$, or $a^{\prime} b_{j}$ outside $b_{j} a c b_{j+1} a^{\prime} c^{\prime}$ to obtain $T^{\prime}$, and that we have to perform a homothety with negative ratio to include $\mathcal{R}^{\prime}$.

Finally, if there are two opposite chords on $b_{j} a c b_{j+1} a^{\prime} c^{\prime}$, say $a c^{\prime}$ and $c a^{\prime}$, we consider two triangulations. Let $T_{1}$ be the one inside the cycle $c^{\prime} b_{j} a$ and let $T_{2}$ be the one obtained from the interior of the 5 -cycle $a c b_{j+1} a^{\prime} c^{\prime}$ by adding the edges $a b_{j+1}$ and $b_{j+1} c^{\prime}$. By the induction we know that $T_{1}$ and $T_{2}$ admit 3-slopes segment representations $\mathcal{R}_{1}$, and $\mathcal{R}_{2}$, and let $\left(s_{a}, s_{b}, s_{c}\right)$ be the shape of $\mathcal{R}_{2}$. We resolve the point by moving the segments as depicted in Figure 10, and the magnitude of each of these moves, except for $\mathbf{b}_{\mathbf{j}}$, is prescribed by the shape $\left(s_{a}, s_{b}, s_{c}\right)$ in order to allow us to copy $\mathcal{R}_{2}$ inside the triangle formed by $\mathbf{a}, \mathbf{b}_{\mathbf{j}+\mathbf{1}}$, and $\mathbf{c}^{\prime}$. Then we shorten $\mathbf{a}$, and $\mathbf{b}_{\mathbf{j}+\mathbf{1}}$ 
to avoid the intersections corresponding to $a b_{j+1}$ and $b_{j+1} c^{\prime}$. The segment $\mathbf{b}_{\mathbf{j}}$ is moved sufficiently to the left to avoid the interior of the triangle containing $\mathcal{R}_{2}$. Then $\mathcal{R}_{1}$ is drawn inside the triangle bordered by $\mathbf{b}_{\mathbf{j}}$, a and $\mathbf{c}^{\prime}$. This is possible because $\mathcal{R}_{2}$ does not intersect this triangle.

Finally note that the moves of $\mathbf{b}_{\mathbf{j}}$ and $\mathbf{b}_{\mathbf{j}+\mathbf{1}}$ are opposite but of proportional magnitudes (up to some constant depending on the shapes $\left(s_{a}, s_{b}, s_{c}\right)$ of $\mathcal{R}^{\prime}$ or $\mathcal{R}_{2}$ ). So it is clear that we can simultaneously move all the segments $b_{\mathbf{j}}$ on $\mathcal{V}$. This concludes the proof of the lemma.

\section{Conclusion}

D. West [19] and H. de Fraysseix and P. Ossona de Mendez [7] independently ask for a generalization of Scheinerman's Conjecture.

Conjecture 1. Planar graphs that are $k$-colorable admit a $k$-slopes segment representation.

The case $k=1$ is trivial. We have seen that the case $k=2$ holds with 2 -slopes contact representations. We have seen that the case $k=3$ also holds. For the final case $k=4$ we would like to apply the same approach as here. This means that we would like to go through a TC-representation of the considered triangulation, and then resolve its degenerate points. There are at least two obstacles for this approach. The first one is to find an order on the degenerate points to resolve them. The left to right approach does not seem sufficient here. The second one is connected to degenerate points $\mathbf{p}$ such that going clockwisely around $\mathbf{p}$ we successively cross segments $\mathbf{a}, \mathbf{b}, \mathbf{c}, \mathbf{d}$, and $\mathbf{a}$ again. In that case its is impossible, restricting ourselves to small pertubations to create a region bordered by $\mathbf{a}, \mathbf{b}, \mathbf{c}$, and $\mathbf{d}$ in the clockwise or counterclockwise order. To avoid this issue we want 4-colorings (i.e. 4-slopes assignements) of planar graphs with particular properties.

Conjecture 2. Planar graphs admit a $\{1,2,3,4\}$-coloring such that ther is no induced $C_{4}$ colored with colors $1,2,3$ and 4 in clockwise order.

Examples show that one cannot extend this condition to non-induced 4-cycles. A positive answer to this conjecture would imply that simple signed planar graphs have chromatic number at most 4, positively answering a conjecture of E. Máčajová, A. Raspaud, and M. Škoviera [15].

Acknowledgements. The author is thankful to Marc de Visme for fruitful discussions on this topic, and to Pascal Ochem for bringing [15] to his attention.

\section{References}

1. N. de Castro, F. Cobos, J.C. Dana, A. Márquez, and M. Noy. Triangle-free planar graphs as segment intersection graphs. J. Graph Algorithms Appl., 6(1):7-26, 2002.

2. J. Chalopin and D. Gonçalves. Every planar graph is the intersection graph of segments in the plane: extended abstract. Proceedings of the forty-first annual ACM symposium on Theory of computing, 631-638, 2009. 
3. J. Czyzowicz, E. Kranakis, and J. Urrutia. A simple proof of the representation of bipartite planar graphs as the contact graphs of orthogonal straight line segments. Inform. Process. Lett., 66(3):125-126, 1998.

4. S. Felsner. Triangle Contact Representations. Midsummer Combinatorial Workshop, 2009.

5. S. Felsner. Rectangle and Square Representations of Planar Graphs. In J. Pach, editor,Thirty Essays on Geometric Graph Theory, Springer, 2013.

6. S. Felsner, H. Schrezenmaier, and R. Steiner. Equiangular Polygon Contact Representations Proc. of WG 2018, LNCS 11159:203-215, 2018. (Long version retrieved at http://page.math.tu-berlin.de/ felsner/Paper/kgons.pdf)

7. H. de Fraysseix and P. Ossona de Mendez. Representations by Contact and Intersection of Segments. Algorithmica, 47:453-463, 2007.

8. H. de Fraysseix, P. Ossona de Mendez, and J. Pach. Representation of planar graphs by segments. Intuitive geometry (Szeged, 1991), Colloq. Math. Soc. János Bolyai, 63:109-117, 1994.

9. D. Gonçalves, L. Isenmann, and C. Pennarun. Planar Graphs as L-intersection or L-contact graphs. Proc. of SODA 2018, 172-184, 2018.

10. D. Gonçalves, B. Lévêque, and A. Pinlou. Triangle Contact Representations and Duality. Proc. of GD 2010, LNCS 6502, 262-273, 2011.

11. I.B.-A. Hartman, I. Newman, R. Ziv. On grid intersection graphs. Discrete Math., 87(1):41$52,1991$.

12. J. Kratochvíl. String graphs. II. Recognizing String Graphs is NP-hard. J. Combin. Theory. Ser. B, 52:67-78, 1991.

13. J. Kratochvíl. Bertinoro Workshop on Graph Drawing, 2007.

14. J. Kratochvíl and J. Matoušek. Intersection Graphs of Segments. J. Combin. Theory. Ser. B, 62:180-181, 1994.

15. E. Máčajová, A. Raspaud, and M. Škoviera. The Chromatic Number of a Signed Graph. Elec. J. of Combinatorics, 23(1): P1.14, 2016.

16. J. Pach and J. Solymosi. Crossing Patterns of Segments. J. Combin. Theory. Ser. A, 96:316325, 2001.

17. E.R. Scheinerman. Intersection classes and multiple intersection parameters of graphs. $P h D$ Thesis, Princeton University, 1984.

18. E.R. Scheinerman. Private communication to D. West.

19. D. West. Open problems. SIAM J. Discrete Math. Newslett., 2(1):10-12, 1991. 